\title{
Measuring the phase variance of light
}

KENNETH L. PREGNELL and DAVID T. PEGG

School of Science, Griffith University, Brisbane 4111, Australia

PACS numbers: 42.50.Dv, 42.50.-p

Author for correspondence: D. T. Pegg

Phone: +61 73875 7152, Fax: + 613875 7656, Email: D.Pegg@sct.gu.edu.au 
Abstract. The results of experiments designed to measure the operational phase cosine and sine variances of weak states of light disagree with the variances predicted by canonical phase formalisms. As these variances are fundamental manifestations of the quantum nature of phase, it is important to be able to measure the canonical variances also. A recent suggestion to do so, based on use of a two-component probe, involves the difficult preparation of exotic states of light which have not yet been produced. In this paper we show how the variances can be measured with simple coherent state inputs. The retrodictive formalism of quantum mechanics provides useful insight into the physics involved. 


\section{Introduction}

While there are some differences in various theoretical quantum descriptions of the phase of light, a common feature is that there should be some uncertainty relation between photon number and phase. Thus the quantum nature of phase should be manifest as an uncertainty, that is as a non-zero variance in the phase probability distribution. This uncertainty should be most pronounced for states of light with very small photon number variances as must pertain, for example, to states that do not differ very much from the vacuum. By contrast, strong coherent states of light, which approximate classical states, should have sharply defined values of phase. For this reason experimental investigations into the quantum nature of the phase of light $[1,2]$ have paid particular attention to finding the width of the phase distribution of states of light with low mean photon number. As the variance of the phase angle $\varphi$ itself depends critically on the $2 \pi$ window assigned to its range of values, such experiments are usually directed at measuring the phase cosine and sine variances $(\Delta \cos \varphi)^{2}$ and $(\Delta \sin \varphi)^{2}$. For small phase variances one might expect from expanding the classical series that $(\Delta \cos \varphi)^{2}+(\Delta \sin \varphi)^{2} \approx(\Delta \varphi)^{2}$. Simple balanced homodyne techniques, sometimes referred to as phase measurements, can be used to obtain a distribution of a suitably defined operational, or measured, sine and cosine of phase [3]. For states with a small enough phase variance, this distribution can give a very good approximation to the canonical phase distribution [4] where the canonical phase is defined as the complement of the photon number operator and can be described mathematically by the formalism in [5]. For weak fields in the quantum regime, however, which by necessity have broader 
phase distributions, significant divergences occur between the canonical and operational phase distributions. This is also true for the operational phase defined and measured by Noh et al. [2] whose distribution width for coherent states has a maximum divergence from that of the canonical distribution for mean photon numbers around unity. More recently other techniques have also been suggested that focus on measuring directly the phase properties of weak fields. These include projection synthesis [6] for measuring the canonical phase distribution and a two-component probe technique [7] for measuring the canonical phase cosine or sine variance. These, however, rely on engineering specifically tailored probe states that, although possible in principle, will be very difficult in practice and have so far not been produced. Even producing the two-component probe of [7] by truncating a coherent state with a quantum scissors device [8] is by no means trivial. Thus on one hand there are techniques that use easily prepared states but which do not measure the canonical phase variances and on the other there are techniques that measure canonical phase variances but rely on exotic quantum states.

In this paper we examine the possibility of measuring the canonical phase cosine and sine variances of optical fields, with a particular interest in weak fields, by using input states which are easily produced in the laboratory, that is, coherent states. We find that, even though the two-component probe states needed for the technique of [7] are effectively not available at present, it is not difficult to use a retrodictive two-component probe state for our purposes. This is a state which, in the less usual retrodictive formalism of quantum mechanics [9], is assigned on the basis of the output of a measurement and which evolves backwards in time from the measurement event. 


\section{Mean of the phase cosine}

By using the hermitian phase operator formalism of [5], in which the operators $\cos \hat{\varphi}$ and $\sin \hat{\varphi}$ commute, we can show that, for a physical state $|c\rangle=\sum c_{n}|n\rangle$, where $|n\rangle$ are photon number states,

$$
\begin{aligned}
& \langle\cos \hat{\varphi}\rangle=\frac{1}{2}\left(\sum_{n} c_{n} c_{n+1}^{*}+\text { c.c. }\right) \\
& \langle\sin \hat{\varphi}\rangle=\frac{i}{2}\left(\sum_{n} c_{n} c_{n+1}^{*}-\text { c.c. }\right)
\end{aligned}
$$

and

$$
\left\langle\cos ^{2} \hat{\varphi}\right\rangle=\frac{1}{2}[1+\langle\cos (2 \hat{\varphi})\rangle]
$$

where

$$
\langle\cos (2 \hat{\varphi})\rangle=\frac{1}{2}\left(\sum_{n} c_{n} c_{n+2}^{*}+\text { c.c. }\right)
$$

The formalism of [5] involves first finding the expectation values in a finite dimensional Hilbert space and then finding the limit of these as the dimensionality of the space is allowed to tend to infinity. A physical state is one with finite energy moments. An alternative method is to use the phase operators acting on Vaccaro’s space [10]. For a physical mixed state, we can find the mean values from the trace of the product of the density operator $\hat{\rho}^{c}$ and the relevant function of the phase operator. We obtain 


$$
\begin{aligned}
& \langle\cos \hat{\varphi}\rangle=\frac{1}{2}\left(\sum_{n} \rho_{n, n+1}^{c}+\text { c.c. }\right) \\
& \langle\sin \hat{\varphi}\rangle=\frac{i}{2}\left(\sum_{n} \rho_{n, n+1}^{c}-\text { c.c. }\right) \\
& \langle\cos (2 \hat{\varphi})\rangle=\frac{1}{2}\left(\sum_{n} \rho_{n, n+2}^{c}+\text { c.c. }\right)
\end{aligned}
$$

The proposed measurement technique uses the beam-splitter arrangement shown in figure 1. A controllable reference field in a coherent state $|\alpha\rangle_{a}=\sum a_{n}|n\rangle_{a}$ is in the input mode $a$ of 50/50 symmetric beam splitter BS1. The state $|c\rangle_{c}$ or $\hat{\rho}^{c}$ to be measured is in the input mode $c$ of beam splitter BS2 and a vacuum state $|0\rangle_{b}$ is in input mode $b$ of BS2. For now we do not specify the transmission and reflection coefficients of BS2. Photon detectors $D_{b}, D_{a}$ and $D_{c}$ are in the output mode $b$ and output mode $a$ of BS1 and in the output mode $c$ of BS2. We shall assume for now that these detectors can count photons with perfect efficiency, no dark counts and negligible dead time. We return to this assumption later.

In the usual predictive formalism of quantum mechanics a density operator $\hat{\rho}$ is assigned to describe the (predictive) state of the field between preparation and measurement based on the outcome of the preparation apparatus. This state evolves forward in time until the field interacts with the measurement apparatus. The measurement apparatus is described in general by a probability operator measure (POM) with elements corresponding to possible outcomes of the measurement [11]. The probability of a measurement outcome is given by the projection of the evolved 
predictive state onto the associated POM element, that is, by the trace of the product of the density operator and the POM element. To avoid unnecessary complications, we assume the predictive states we assign to the input fields of the device in figure 1 describe the fields at their entry to the beam splitters. The free evolution in the intermediate mode $b$ between the beam splitters only changes the phase of the field in this mode so, by choosing the distance between beam splitters to be an integer number of wavelengths, we can ignore this evolution. In practice, even if this is not the case such a phase shift can be compensated by adjusting the phase of $|\alpha\rangle_{a}$. Finally we can ignore the free evolution in all the output modes, as these do not affect the photocount probabilities. We denote the (forward time) unitary operator for the actions of beam splitters BS2 and BS1 as respectively $\hat{R}_{2}$, which acts on states in modes $c$ and $b$, and $\hat{R}_{1}$ which acts on states in modes $a$ and $b$.

The initial combined density operator for the three input fields is

$$
\hat{\rho}=\hat{\rho}^{c} \otimes|0\rangle_{b}{ }_{b}\langle 0|\otimes| \alpha\rangle_{a}{ }_{a}\langle\alpha|
$$

and the POM element for the detection of $N, n_{b}$ and $n_{a}$ photons in output modes $c, b$ and $a$ respectively is

$$
\begin{aligned}
\hat{\Pi}\left(N, n_{b}, n_{a}\right) & =\hat{\Pi}_{c} \otimes \hat{\Pi}_{b} \otimes \hat{\Pi}_{a} \\
& =|N\rangle_{c}{ }_{c}\left\langle N|\otimes| n_{b}\right\rangle_{b}{ }_{b}\left\langle n_{b}|\otimes| n_{a}\right\rangle_{a}{ }_{a}\left\langle n_{a}\right|
\end{aligned}
$$


The probability for the detection of $N, n_{b}$ and $n_{a}$ photons in output modes $c, b$ and $a$ respectively is then

$$
P\left(N, n_{b}, n_{a}\right)=\operatorname{Tr}_{a b c}\left[\hat{R}_{1} \hat{R}_{2} \hat{\rho} \hat{R}_{2}^{\dagger} \hat{R}_{1}^{\dagger} \hat{\Pi}\left(N, n_{b}, n_{a}\right)\right]
$$

Substituting from (8) and (9) and using the cyclic property of the trace we can rewrite this as

$$
P\left(N, n_{b}, n_{a}\right)=\operatorname{Tr}_{c}\left[\hat{\rho}^{c} \hat{\Pi}^{\mathrm{retr}}\left(N, n_{b}, n_{a}\right)\right]
$$

Here $\hat{\Pi}^{\text {retr }}\left(N, n_{b}, n_{a}\right)$ is the retrodictive POM element:

$$
\begin{aligned}
\hat{\Pi}^{\mathrm{retr}}\left(N, n_{b}, n_{a}\right) & ={ }_{b}\left\langle 0\left|\hat{R}_{2}^{\dagger} \hat{\Pi}_{c} \otimes{ }_{a}\left\langle\alpha\left|\hat{R}_{1}^{\dagger} \hat{\Pi}_{b} \otimes \hat{\Pi}_{a} \hat{R}_{1}\right| \alpha\right\rangle_{a} \hat{R}_{2}\right| 0\right\rangle_{b} . \\
& =|r\rangle_{c c}\langle r|
\end{aligned}
$$

where, from (9),

$$
|r\rangle_{c}={ }_{b}\left\langle 0\left|\hat{R}_{2}^{\dagger}\right| N\right\rangle_{c a}\left\langle\alpha\left|\hat{R}_{1}^{\dagger}\right| n_{b}\right\rangle_{b}\left|n_{a}\right\rangle_{a} .
$$

The state $|r\rangle_{c}$ in (13) can be interpreted as an unnormalised retrodictive state of the field in input mode $c$ associated with the measurement outcome of $N, n_{b}$ and $n_{a}$ photons being detected. In the usual predictive formalism the state of a system between 
preparation and measurement is assigned on the basis of the preparation outcome. In the retrodictive formalism this state is assigned on the basis of the outcome of the measurement, specifically $\hat{\rho}^{\text {retr }}=\hat{\Pi}_{j} / \operatorname{Tr} \hat{\Pi}_{j}$ where $\hat{\Pi}_{j}$ is the POM element for the particular outcome $j$ [12], and then evolves backwards in time from the measurement event to the preparation event. In our case the retrodictive fields associated with the measurement outcomes of the photon detectors are simple number state projectors which are already normalised. We can see from (13) that the retrodictive fields $\left|n_{b}\right\rangle_{b}$ and $\left|n_{a}\right\rangle_{a}$ associated with the measurements in the output mode $b$ and output mode $a$ evolve backwards in time and are entangled by means of beam splitter BS1. This entangled state is projected onto $|\alpha\rangle_{a}$ to yield an unnormalised retrodictive probe state

$$
|q\rangle_{b}={ }_{a}\left\langle\alpha\left|\hat{R}_{1}^{\dagger}\right| n_{b}\right\rangle_{b}\left|n_{a}\right\rangle_{a}
$$

in the intermediate mode $b$, that is, between the two beam splitters. As we shall see later this is a retrodictive two-component state which performs a similar function to the predictive two-component probe of [7]. The state $|q\rangle_{b}$ is entangled by beam splitter BS2 with the retrodictive state from the measurement outcome of the detector $D_{c}$. This state in turn is projected onto the vacuum in input mode $b$ to give the retrodictive state $|r\rangle_{c}$ for projection onto the state to be measured. We remark here that if the state $|c\rangle_{c}$ is a coherent state then in the predictive picture there is no entanglement at all because all input states are coherent. The entanglement mentioned above occurs in retrodiction. In addition to giving new insight, working in terms of the retrodictive probe state (13) has 
practical calculational advantages in our case in that the fields that evolve backwards originate from single photon number states associated with the measurement outcomes. The simplest possible retrodictive probe is associated with $n_{a}=n_{b}=0$. In this case we find that the retrodictive probe state $|q\rangle_{b}$ is just the vacuum and so $|r\rangle_{c}$ is just proportional to $|N\rangle_{c}$. Thus only the diagonal matrix elements of $\hat{\rho}^{c}$ are obtainable from the measured probabilities. The next simplest retrodictive probes are associated with the measurement result $n_{a}=0, n_{b}=1$ and $n_{a}=1, n_{b}=0$. For a symmetric beam splitter with transmission coefficient $\cos \theta$ we have the relations (for a general description of the beam splitter see, for example, [13])

$$
\begin{aligned}
& \hat{R}^{\dagger} \hat{a}^{\dagger} \hat{R}=\hat{a}^{\dagger} \cos \theta-i \hat{b}^{\dagger} \sin \theta \\
& \hat{R}^{\dagger} \hat{b}^{\dagger} \hat{R}=\hat{b}^{\dagger} \cos \theta-i \hat{a}^{\dagger} \sin \theta \\
& \hat{R}^{\dagger}|0\rangle_{b}|0\rangle_{a}=|0\rangle_{b}|0\rangle_{a}
\end{aligned}
$$

with $\hat{a}^{\dagger}, \hat{b}^{\dagger}$ and $\hat{c}^{\dagger}$ the creation operators for modes $a, b$ and $c$ respectively. For a 50/50 beam splitter $\theta=\pi / 4$. Using this value for BS1we easily find, by writing $|1\rangle_{b}=\hat{b}^{\dagger}|0\rangle_{b}$, the unnormalised retrodictive probe state for $n_{a}=0, n_{b}=1$ to be

$$
|q\rangle_{b}=\left(a_{o}^{*}|1\rangle_{b}-i a_{1}^{*}|0\rangle_{b}\right) / \sqrt{2}
$$

This two-component probe can be used to obtain information about the offdiagonal matrix elements of $\hat{\rho}^{c}$ as follows. Substituting into (13) allows us to find $|r\rangle_{c}$ 
by writing $|N\rangle_{c}=\hat{c}^{\dagger N}|0\rangle_{c} / \sqrt{N !}$ and using (15) and (16) with $\hat{c}^{\dagger}$ in place of $\hat{a}^{\dagger}$. We find eventually

$$
|r\rangle_{c}=-i\left(\cos ^{N} \theta\right)\left[a_{1}^{*}|N\rangle_{c}+(\sin \theta) \sqrt{N+1} a_{0}^{*}|N+1\rangle_{c}\right] / \sqrt{2}
$$

which allows us to find the retrodictive POM element from (12) and hence, from (11), the measurable probability

$$
\begin{gathered}
P(N, 1,0)=\left(\frac{1}{2} \cos ^{2 N} \theta\right)\left[\left|a_{1}\right|^{2} \rho_{N, N}^{c}+\left(\sin ^{2} \theta\right)(N+1)\left|a_{0}\right|^{2} \rho_{N+1, N+1}^{c}\right. \\
\left.+\left(a_{0} a_{1}^{*} \sqrt{N+1} \rho_{N+1, N}^{c} \sin \theta+\text { c.c. }\right)\right]
\end{gathered}
$$

For now we do not specify the value of $\theta$ for BS2.

Similarly we find the probability for $n_{a}=1, n_{b}=0$ and $N$ counts in detector $D_{c}$ to be

$$
\begin{gathered}
P(N, 0,1)=\left(\frac{1}{2} \cos ^{2 N} \theta\right)\left[\left|a_{1}\right|^{2} \rho_{N, N}^{c}+\left(\sin ^{2} \theta\right)(N+1)\left|a_{0}\right|^{2} \rho_{N+1, N+1}^{c}\right. \\
\left.-\left(a_{0} a_{1}^{*} \sqrt{N+1} \rho_{N+1, N}^{c} \sin \theta+\text { c.c. }\right)\right]
\end{gathered}
$$

For the first experiment we choose the phase of the coherent reference state $|\alpha\rangle_{a}$ so that $a_{n}$ are all real and positive. From (20), (21) and (5) an expression for the mean of the phase cosine in terms of the measurable probabilities can then be written as 


$$
\langle\cos \hat{\varphi}\rangle=\sum_{N} \frac{P_{1}(N, 1,0)-P_{1}(N, 0,1)}{2\left(\cos ^{2 N} \theta\right)\left|a_{0} a_{1}\right| \sqrt{N+1} \sin \theta}
$$

where the subscript on the probability refers to the first experiment.

\section{Variance of the phase cosine and sine}

The next simplest possible retrodictive probe originates from the measurement event $n_{a}=n_{b}=1$. Again for the 50/50 beam splitter BS1 we have $\theta=\pi / 4$. Writing $|1\rangle_{b}=\hat{b}^{\dagger}|0\rangle_{b}$ and $|1\rangle_{a}=\hat{a}^{\dagger}|0\rangle_{a}$ we obtain from (15), (16) and (17), the two-component retrodictive probe state

$$
|q\rangle_{b}=-i\left(a_{o}^{*}|2\rangle_{b}+a_{2}^{*}|0\rangle_{b}\right) / \sqrt{2}
$$

leading to

$$
|r\rangle_{c}=-i\left(\cos ^{N} \theta\right)\left[a_{2}^{*}|N\rangle_{c}-a_{0}^{*}\left(\sin ^{2} \theta\right) \sqrt{(N+1)(N+2) / 2}|N+2\rangle_{c}\right] / \sqrt{2} \quad .
$$

From (11) and (12), this gives the probability

$$
\begin{gathered}
P(N, 1,1)=\left(\frac{1}{2} \cos ^{2 N} \theta\right)\left[\left|a_{2}\right|^{2} \rho_{N, N}^{c}+\frac{1}{2}\left(\sin ^{4} \theta\right)(N+1)(N+2)\left|a_{0}\right|^{2} \rho_{N+2, N+2}^{c}\right. \\
\left.-\left(a_{0} a_{2}^{*} \sqrt{(N+1)(N+2) / 2} \rho_{N+2, N}^{c} \sin ^{2} \theta+\text { c.c. }\right)\right]
\end{gathered}
$$


To allow the experiment with this probe to be conducted simultaneously with the experiment to find $\langle\cos \hat{\varphi}\rangle$, we take $a_{0}$ and $a_{2}$ to be real and positive and find $P_{1}(N, 1,1)$ by simply replacing $a_{0} a_{2}^{*}$ in (25) by $\left|a_{0} a_{2}\right|$.

After measuring the probabilities $P_{1}(N, 1,0), P_{1}(N, 0,1)$ and $P_{1}(N, 1,1)$ the experiment is repeated with a phase shift of $\pi / 2$ in the reference state $|\alpha\rangle_{a}$, which has the effect of changing $a_{n}$ to $a_{n} \exp (i n \pi / 2)$. Thus now $a_{0}=\left|a_{0}\right|, a_{1}=i\left|a_{1}\right|$, and $a_{2}=-\left|a_{2}\right|$ in (20), (21) and (25), yielding $P_{2}(N, 1,0), P_{2}(N, 0,1)$ and $P_{2}(N, 1,1)$. From (6) we can then obtain the mean phase sine from the measured results as

$$
\langle\sin \hat{\varphi}\rangle=\sum_{N} \frac{P_{2}(N, 1,0)-P_{2}(N, 0,1)}{2\left(\cos ^{2 N} \theta\right)\left|a_{0} a_{1}\right| \sqrt{N+1} \sin \theta}
$$

We also find from (7) that

$$
\langle\cos (2 \hat{\varphi})\rangle=\sum_{n} \frac{P_{2}(N, 1,1)-P_{1}(N, 1,1)}{2\left|a_{0} a_{2}\right| \sqrt{(N+1)(N+2) / 2} \cos ^{2 N} \theta \sin ^{2} \theta}
$$

After these values are obtained from the measured probabilities, the mean square phase cosine can be found from (3) and finally the phase cosine variance calculated as $\left\langle\cos ^{2} \hat{\varphi}\right\rangle-\langle\cos \hat{\varphi}\rangle^{2}$. Further, we can also write from the phase formalism of [5] 


$$
\left\langle\sin ^{2} \hat{\varphi}\right\rangle=\frac{1}{2}[1-\langle\cos (2 \hat{\varphi})\rangle]
$$

which allows us also to find the phase sine variance from the measured probabilities.

\section{Discussion}

We have already assigned a value to the phase of the reference state $|\alpha\rangle_{a}$ but still have freedom to choose its mean photon number $|\alpha|^{2}$. To avoid quotients of very small numbers, it is worth maximising the denominator, and hence the numerator, in (22) and (26). Thus we should choose a reference state to maximise $\left|a_{0} a_{1}\right|$ and for (25) we should maximise $\left|a_{0} a_{2}\right|$. The former and latter are maximised for mean photon numbers of 0.5 and 1 respectively. The experiment could in principle be run for both these values but in practice it would be simpler just use a compromise value between 0.5 and 1 .

We have yet to choose the reflection to transmission ratio of the beam splitter BS2. Again it is useful to choose a ratio which maximises the denominators of the terms in (22). The optimum value of $\sin \theta$ for each term is $(1+2 N)^{-1 / 2}$. For (27) the optimum value of $\sin \theta$ for each term is $(1+N)^{-1 / 2}$. If necessary the experiment could be repeated for different values of $N$ but, given we are mainly interested in weak fields, the spread in values of $N$ should not be huge. Thus a compromise value of around $\sin \theta \approx(1+<n>)^{-1 / 2}$ should be adequate for determining both (22) and (27), where $<n>$ is the mean photon number of the field to be measured. Thus for fields with a mean photon number around unity a 50/50 beam splitter would be quite suitable. For stronger fields an increase in the transmission of BS2 would be desirable. 
Once we have measured $\langle\cos \hat{\varphi}\rangle$ and $\langle\sin \hat{\varphi}\rangle$ we can easily find $\langle\exp (i \hat{\varphi})\rangle$ which, for a pure state $|c\rangle=\sum c_{n}|n\rangle$, is just $c_{n}^{*} c_{n+1}$. Then, if the state to be measured is a pure state with no gaps in the photon number distribution, we can use these results to reconstruct the complete state, as shown in [7]. If there are gaps then, provided these only involve the vanishing of a single number state coefficient, the complete state could still be reconstructed using a knowledge of $\langle\exp (i 2 \hat{\varphi})\rangle$ to bridge the gaps [7]. This would require an additional measurement of $\langle\sin (2 \hat{\varphi})\rangle$ which we could obtain by repeating the measurement of $\langle\cos (2 \hat{\varphi})\rangle$ with a phase shift of $\pi / 4$ applied to the measured field.

The detection probabilities used above are those that would be obtained by perfect detectors. Practical photodetectors suffer from non-unit efficiencies that reduce the number of counts, the presence of dark counts and a non-zero dead time following a count during which no other counts are registered. Using weak fields, in which we are particularly interested, and sufficiently long gating times reduces the effect of the dead time. Other techniques can also be used for this purpose [14]. For known efficiency and dark count rate the ideal photocount probabilities can be found from the statistics of the experiment. The probability that ideal detectors would have detected $N, n_{b}$ and $n_{a}$ photons in output modes $c, b$ and $a$ respectively is

$$
P\left(N, n_{b}, n_{a}\right)=\sum_{M, m_{b}, m_{c}} P\left(M, m_{b}, m_{a}\right) p(N \mid M) p\left(n_{b} \mid m_{b}\right) p\left(n_{a} \mid m_{a}\right)
$$


where $M, m_{b}$ and $m_{a}$ are the actual counts in the detectors and $p(N \mid M)$, for example, is the probability that an ideal detector would have counted $N$ photons if the actual detector counted $M$ photons. An expression for $p(N \mid M)$ for non-unit efficiency can be derived using Bernouilli transforms as in, for example, [15-17]. Allowance for dark counts can also be incorporated using the techniques, for example, in $[8,17]$.

\section{Conclusion}

The measurement procedure described here can be used for the complete reconstruction of reproducible pure states of light which have no gaps in their number state distributions. For a mixed state the diagonal, nearest off-diagonal and the next to nearest off-diagonal matrix elements can be measured. These determine the mean sine and cosine of the phase as well as the mean square of the sine and cosine, from which the variance of the phase sine and cosine can be found.

The above quantities can also be obtained by means of a two-component probe field technique suggested in [7]. There are very important differences however. In [7], where states are assigned to the probe fields according to the usual predictive quantum formalism, the required probe states are, as acknowledged in that paper, very difficult to prepare. Indeed, to our knowledge, such two-component states have yet to be produced. The preparation method suggested for [7] was optical truncation using quantum scissors [8], so the measurement would require three beam splitters in all, with separate experiments being run with each different probe. More seriously, the preparation of the probe in a one-photon and vacuum superposition in [7] requires the injection of a single 
photon state into one input port and the probe in a two-photon and vacuum superposition requires the simultaneous injection of a single photon into two input ports.

In contrast to the technique suggested in [7], the method proposed here has real practical advantages. Only two beam splitters are used and, apart from the state to be measured, the only states injected into other input ports are vacuum and coherent states. These are not only considerably easier to prepare, their coherence lengths can allow longer gating times, reducing the effect of dead times. The retrodictive one-photon states needed to construct the retrodictive probe originate from photon detection events and are thus more readily available than their predictive counterparts which originate from preparation events. Further, because the retrodictive probe states are produced by the detection events, all three probe states, including the retrodictive vacuum state used for measuring the diagonal density matrix elements, are produced in the one experiment. There is no need to run separate experiments for different probe states.

In addition to the improved experimental practicality of the proposal in this paper, we feel that it also has importance as an application of the little used but completely rigorous retrodictive formalism [9]. Until recently this formalism has been treated as having a philosophic interest only but is beginning to find use in quantum communication problems [18] where the receiver and measurer of a quantum state must retrodict what state was prepared and sent. The formalism was also helpful in examining, amongst other problems, the quantum scissors device for truncation optical states [8]. For the present paper we deliberately sought two-component retrodictive probes mirroring the probes of [7]. To emphasise that the retrodictive formalism yields the same measurable probabilities as the predictive formalism we used the standard predictive picture in (10) 
to begin our mathematical analysis. It is interesting, however, to compare our approach with the retrodictive analysis of the quantum scissors [8]. In the retrodictive picture, the state to be truncated is in one input mode of a beam splitter with detectors in the two output modes. When one of these detects one photon and the other detects zero photons, the retrodictive state in the other input mode is a superposition of the vacuum and one photon states, so the actual cutting out of the higher photon state components is done at this beam splitter. The other beam splitter of the quantum scissors creates a predictive entangled state. Projection of the retrodictive state onto this state effectively converts the retrodictive state into a predictive state with the coefficients of the vacuum and one photon components interchanged. The beam splitter BS1 of the present paper can be regarded as the part of the quantum scissors that creates the retrodictive two-component state. As we wish to use this retrodictive probe directly, there is no need to employ another beam splitter to convert it to a predictive probe. This also dispenses with the necessity to produce and inject single-photon fields.

Finally, the insight provided by the retrodictive formalism of quantum mechanics has enabled us to propose a relatively simple experiment to measure some of the canonical phase properties of light. There is now less need to define separate operational phase properties based on easily performed experiments.

\section{Acknowledgments}

DTP thanks the Australian Research Council for financial support.

\section{References}


[1] Gerhardt, H., Welling, H. and Frolich, D 1973, Appl. Phys., 2, 91; Gerhardt, H., Büchler, U. and Litfin, G., 1974, Phys. Lett. A, 49, 119.

[2] Noh, J. W., Fougères, A., and Mandel, L.,1991, Phys. Rev. Lett. 67, 1426; Noh, J. W., Fougères, A., and Mandel, L.,1992, Phys. Rev. A 45, 424; Noh, J. W., Fougères, A., and Mandel, L.,1992, Phys. Rev. A 46, 2840; Noh, J. W., Fougères, A., and Mandel, L.,1993, Phys. Rev. Lett. 71, 2579.

[3] Barnett, S. M. and Pegg, D. T., 1986, J. Phys. A, 19, 3849.

[4] Vaccaro, J. A. and Pegg, D. T., 1994, Optics Commun., 105, 335.

[5] Pegg, D. T. and Barnett, S. M., 1988, Europhys. Lett. 6, 483; Barnett, S.M. and Pegg, D. T., 1989, J. mod. Optics 36, 7; Pegg, D. T. and Barnett, S. M., 1989, Phys. Rev. A, 39, 1665.

[6] Barnett, S. M. and Pegg, D. T., 1996, Phys. Rev. Lett., 76, 4148; Pegg, D. T., Barnett, S. M. and Phillips, L. S., 1997, J. mod. Optics, 44, 2135.

[7] Pegg, D. T. and Barnett S. M., 1999, J. mod. Optics, 46, 981.

[8] Pegg, D. T., Phillips, L. S. and Barnett, S.M. 1998, Phys. Rev. Lett., 81,1604; Barnett, S. M. and Pegg, D. T., 1999, Phys. Rev. A, 60, 4965.

[9] Aharonov, Y., Bergman, P. G. and Lebowitz, J. L., Phys. Rev. 134, B1410 (1964); Penfield, R. H., Am. J. Phys. 34, 422 (1966); Aharonov, Y. and Albert, D. Z., Phys. Rev. D 29, 223 (1984); Aharonov Y. and Albert, D. Z., Phys. Rev. D 29, 228 (1984); Aharonov, Y. and Vaidman, L., J. Phys. A: Math. Gen. 24, 2315 (1991); Pegg D. T. and Barnett, S. M., Quantum and Semiclass. Opt. 1, 442 (1999); Barnett, S. M., Pegg, D. T., Jeffers, J. and Jedrkiewicz, O., J. Phys. B: At. Mol. Opt. Phys. 33, 3047 (2000). 
[10] Vaccaro, J. A., 1995, Phys. Rev. A, 51, 3309.

[11] Helstrom, C. W. 1976 Quantum Detection and Estimation Theory (New York: Academic Press)

[12] Barnett, S. M., Pegg, D. T. and Jeffers, J. 2000, J. mod. Optics 47, 1779.

[13] Barnett, S. M. and Radmore, P. M., 1997, Methods in Theoretical Quantum Optics (Oxford: Oxford University Press), p. 84.

[14] Paul, H., Torma, P., Kiss, T. and Jex, I., 1997, Phys. Rev. Lett. 76, 2464.

[15] Lee, C. T., 1993, Phys. Rev. A 48, 2285.

[16] Pegg, D. T. and Barnett S. M., 1999, J. mod. Optics, 46, 1657.

[17] Barnett, S. M., Phillips, L. S. and Pegg, D. T., 1998 Optics Commun., 158, 45.

[18] Barnett, S. M., Pegg, D. T., Jeffers, J., Jedrkiewicz, O. and Loudon, R. Phys. Rev. A 62, 022313 (2000). 


\section{Caption to figure}

Figure 1. Apparatus for measuring the phase cosine and sine variance. The horizontal mode is $b$ and the vertical modes are $c$ and $a$. The state $|c\rangle_{c}$ or $\hat{\rho}^{c}$ to be measured is in the input mode $c$ of beam splitter BS2 and a vacuum state $|0\rangle_{b}$ is in input mode $b$ of BS2. A reference field in a coherent state $|\alpha\rangle_{a}$ is in the input mode $a$ of 50/50 symmetric beam splitter BS1. Photon detectors $D_{b}, D_{a}$ and $D_{c}$ are in the output modes $b, a$ and $c$.

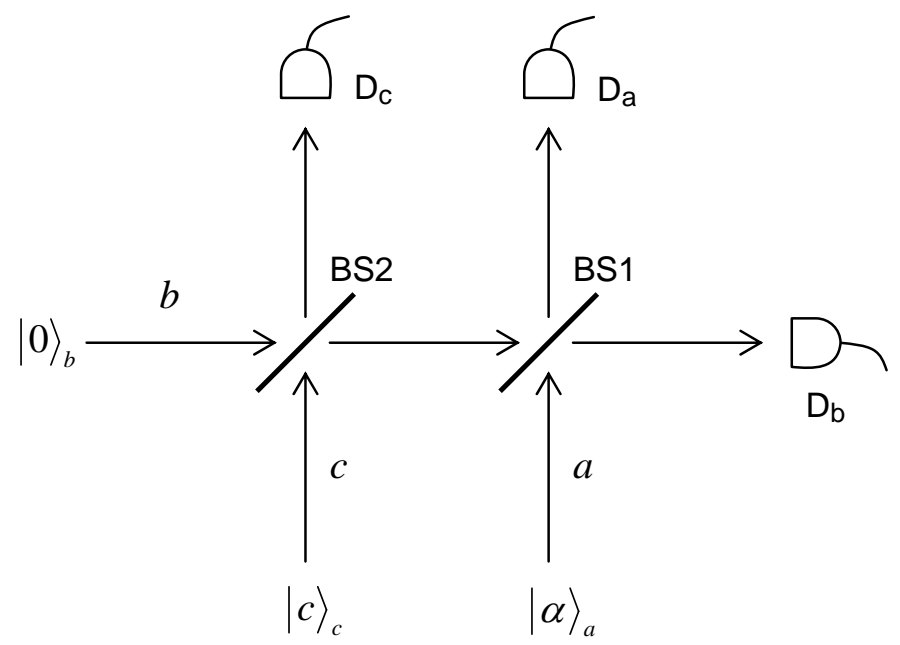

